\title{
Commentary about open-label randomized controlled study of ivermectin in mild to moderate COVID-19
}

\section{To the Editor:}

We have carefully read the article from Podder et al. [1] published on July 2020 at this journal (volume 14, issue 2). In this regard, the authors mentioned approval of their study by the director of the health center, but not a methodological and ethical evaluation by an institutional board. We noted that the trial is not registered in ClinicalTrials.gov, unlike the trial of e.g. Chowdhury et al. [2] from the same country (cited in the article). A board would have questioned the low statistical power of the design, as it only had 62 subjects. We consider inappropriate the choice of a negative outcome of 10-day RT-PCR test as a result in outpatients, since it already was not recommended by the WHO in June 2020 [3].

The authors excluded subjects taking hydroxychloroquine or antimicrobials other than doxycycline, which could introduce considerable selection bias because it ruled out $80 \%$ of patients with a positive RT-PCR test. Lack of concealment of the randomization sequence and systematic allocation were additional factors of bias when distributing patients to the study groups. On the other hand, the lack of blinding increased the risk of information bias, since the outcome is the absence of symptoms. But the most objectionable methodological element was the exclusion of 20 subjects after allocation, based on criteria of lack of information and time of symptoms greater than seven days, not previously defined as study exclusion criteria.

We consider inadequate that the authors combined mild and moderate cases of the disease and managed both categories in a single study of outpatients, when moderate cases probably must be hospitalized or receive stricter monitoring. Since May 2020, WHO advised against the decision to administer antibiotics (e.g. doxycycline) to patients without evidence of bacterial pneumonia [4].
When calculating the coefficients of variation of the times until the patients' recovery, high variability is found in almost all described symptoms durations. We would recommend to the authors to perform a normality contrast test (e.g. Shapiro-Wilk) to check the relevance of the t-test, due to a possible lack of a normal distribution of the data. Even for smaller subgroups (about 6 patients per group with dyspnea or fatigue) the potential utility of a test of difference of medians (e.g. Mann-Whitney $U$ test) had to be evaluated.

\section{References}

1. Podder CS, Chowdhury N, Sina MI, Haque WMMU. Outcome of ivermectin treated mild to moderate COVID-19 cases: a single-centre, open-label, randomised controlled study. IMC J Med Sci. 2020; 14(2).

2. Chowdhury ATMM, Shahbaz M, Karim MR, Islam J, Guo D, He S. A randomized trial of ivermectin-doxycycline and hydroxychloroquineazithromycin therapy on COVID19 patients. Research Square; 2020.

3. World Health Organization. Criteria for releasing COVID-19 patients from isolation: scientific brief: June 17, 2020. Geneva: World Health Organization; 2020. 5 p. Report No.: WHO/2019-nCoV/Sci_Brief/Discharge_From_ Isolation/2020.1.

4. World Health Organization. Clinical management of COVID-19: interim guidance, 27 May 2020. Geneva: World Health Organization; 2020. 62 p. Report No.: WHO/2019-nCoV/clinical/2020.5.

Eduardo Ortega-Guillén, MSc

Hospital Nacional Alberto Sabogal SologurenEsSalud, Callao, Peru

Universidad Nacional Mayor de San Marcos, Lima, Peru.

*Corresponding author: Giovanni Meneses, Departamento Académico de Medicina Preventivay Salud Pública, Facultad de Medicina, Universidad Nacional Mayor de San Marcos, Lima, Peru. ZIP Code: 15307. E-mail: gmenesesf@unmsm.edu.pe 
Giovanni Meneses*, PhD

Hospital San Juan de Lurigancho, San Juan de Lurigancho, Lima, Peru

Universidad Nacional Mayor de San Marcos, Lima, Peru.

\section{*Corresponding author}

[Editor's note: Reference \# 5 is deleted as the reference is missing/not cited within the text.]

\section{Authors' reply}

We appreciate Eduardo Ortega-Guillén, MSc and Giovanni Meneses, PhD for the issues they raised about our study. With all admiration to their concerns, our responses to disavow most of the claims are that the study was performed in a resource-limited primary healthcare setting, where a well-organized institutional review board is not a reality. The local health complex authority consisting of Head of the Upazila health complex and other senior consultants decide and approve conduction of any study at the center. The authority assesses the scientific and ethical aspects of the study(s). This study was also approved by the same body. Though not mandatory - it would have been better if the study was registered in ClinicalTrials.gov site. At the beginning of the study repeat RT-PCR for end of isolation was recommended [1]. We excluded subjects taking hydroxychloroquine or antimicrobials other than doxycycline to eliminate confounders and determine the optimum benefit of ivermectin over the usual care. We also excluded the patients presented after one week or more as ivermectin was presumed to be effective if initiated early in the disease. Though antibiotics are not recommended in COVID management if there is no bacterial infection suspected; it was the centre's protocol to treat every suspected patient of pneumonia with doxycycline at presentation as there were not sufficient investigation facilities to exclude community-acquired pneumonia. In our national and WHO interim guidelines, patients with the mild and moderate disease are advised to be managed at home [1,2]. In some cases, mild and moderate cases are clinically overlapped, so we managed these patients on outdoor patient department (OPD) basis, preserving the valuable hospital beds for more severe cases. Regarding the relevance of t-test, yes, we agree with Eduardo Ortega-Guillén and Giovanni Meneses that it would have been better if we used a nonparametric test for some cases to find out the differences of medians. As suggested, we have re-analyzed our data by Shapiro-Wilk and Mann-Whitney tests, but the results remained as before and there was no change of significance. Also, similar to our observations, several studies showed no conclusive benefits after ivermectin use [3].

\section{References}

1. World Health Organization. Clinical management of COVID-19: interim guidance, 27 May 2020. Geneva: World Health Organization; 2020. 62 p. Report No.: WHO/2019-nCoV/clinical/2020.5.

2. Disease Control Division. Directorate General of Health Services. Ministry of Health \& Family Welfare. Government of the People's Republic of Bangladesh. National Guidelines on clinical management of coronavirus disease 2019 (COVID-19). Bangladesh: Directorate General of Health Services, Ministry of Health \& Family Welfare; 2020. Version 7.0.

3. National Institutes of Health. COVID-19 treatment guidelines panel. Coronavirus disease 2019 (COVID-19) treatment guidelines. [accessed on February 25, 2021]; Available from: https://www.covid19treatmentguidelines.nih.g ov/.

Wasim Md Mohosin Ul Haque* Department of Nephrology, BIRDEM General Hospital 122 Kazi Nazrul Islam Avenue, Dhaka 1000, Bangladesh; Email: wmmhaque@live.com

Chinmay Saha Podder

Debidwar Upazila Health Complex

Debidwar, Cumilla, Bangladesh.

\section{*Corresponding author}

